\title{
The Value of Biological Image Technology in Improving Athletes' Motor Skills
}

\author{
Lu Huang $\mathbb{D}^{1}$ and Hongfei Han (D) $^{2}$ \\ ${ }^{1}$ Weinan Normal University, Weinan, Shaanxi 714000, China \\ ${ }^{2}$ Weinan Youth Sports School, Weinan, Shaanxi 714000, China \\ Correspondence should be addressed to Lu Huang; 3080104131@caa.edu.cn
}

Received 22 June 2021; Accepted 18 August 2021; Published 14 September 2021

Academic Editor: Gustavo Ramirez

Copyright $\odot 2021$ Lu Huang and Hongfei Han. This is an open access article distributed under the Creative Commons Attribution License, which permits unrestricted use, distribution, and reproduction in any medium, provided the original work is properly cited.

\begin{abstract}
Objective. To explore the value of biological image technology in improving athletes' motor skills. Methods. Golfers were given biofeedback relaxation training, and the variation trend and influence of biofeedback training on athletes' heart rate variability were explored by monitoring and feedback evaluation of athletes' heart rate variability. Results. Biofeedback relaxation training can help athletes improve the balance of sympathetic nerve and parasympathetic nerve, effectively inhibit the activity of sympathetic nerve, enhance the tension, effectively increase the heart rate variability of athletes, and strengthen the ability of psychological relaxation. Conclusion. Part of the research results show that biofeedback training can enhance athletes' athletic performance and improve their athletic performance to a certain extent.
\end{abstract}

\section{Introduction}

In recent years, biofeedback relaxation training has been gradually applied to the field of sports. There are a large number of studies on testing athletes using heart rate variability in China. Most biofeedback training only carries out preliminary short-term experiments on athletes by regulating athletes' sympathetic and parasympathetic activities. It is made in a balanced state to enhance the self-coordination ability of athletes, reduce the tension of athletes, and give full play to their own technical and tactical level [1]. Researchers can use the measured heart rate variability index of accurate understanding of the athletes' physical and psychological state through the appropriate way to improve the athletes' heart rate variability; some research results present that biofeedback training can enhance athletic performance of athletes to some extent to improve the performance of athletes [2]. However, the research process is too short or monotonous, with small amount of data, errors in the results, and certain boredom of some subjects. Some research results show that biofeedback training can enhance athletic performance and improve athletic performance of athletes to a certain extent.

\section{Experimental Subjects and Data}

2.1. Biofeedback. See Figure 1 for the biological feedback flow chart. The research of biofeedback covers a wide range of fields and plays a positive role in sports psychology in particular. With the repeated use of biofeedback instrument, the human body's direct perception of the information in the body is gradually deepened, and the sensitivity is gradually improved. Finally, the indirect perception of the body is transformed into direct perception. At this time, if we pause perception and continue to use the biofeedback instrument, we can still obtain a good relaxation effect, which further shows that the biofeedback instrument is an excellent tool for relaxation $[3,4]$. With the continuous development of sports psychology, some advanced electronic equipment have been gradually introduced into the training of athletes' psychological function. As an important behavioral intervention method, biofeedback can improve athletes' self-psychological control ability, psychological balance ability, and anti-interference ability, accelerate the recovery of sports fatigue, and promote the development of athletes' performance ability and competitive ability in competitions. 


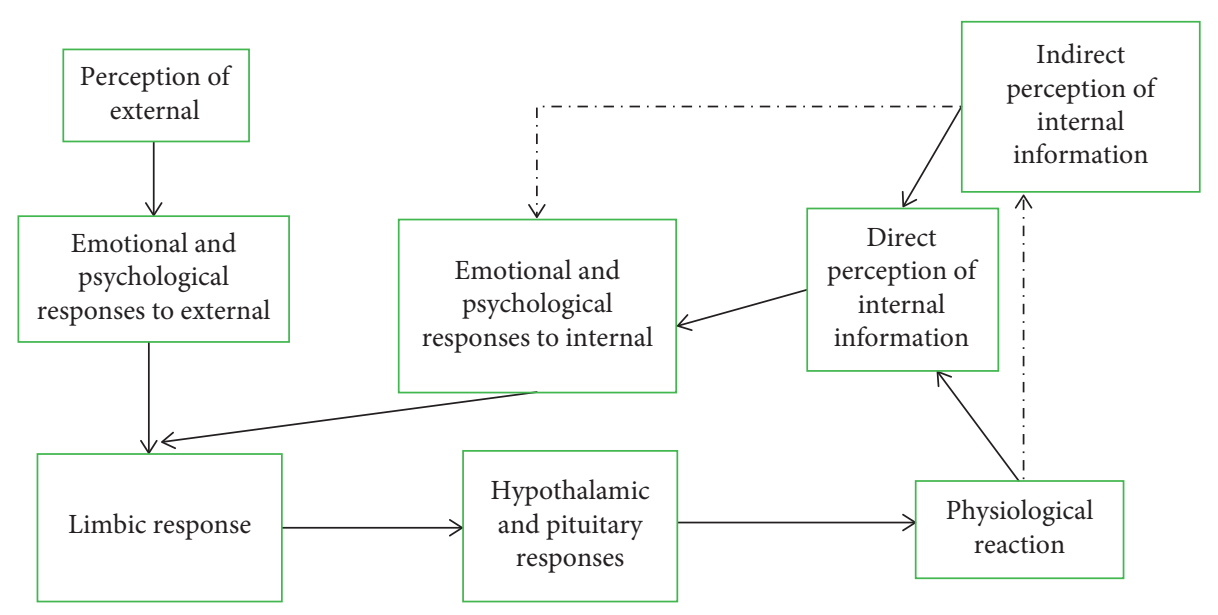

FIGURE 1: Biofeedback.

2.2. Golfers. The golf team of Jiangxi Information Application Vocational and Technical College consists of 10 athletes, 6 male athletes and 4 female athletes (average age: $19.00 \pm 0.99$ years old). In the end, 9 participants (the average age of six male athletes and three female athletes is $19.88 \pm$ 1.05 years old. The average height of male athletes is $1.75 \pm$ 0.03 meters.) were selected as available data. Average height for women $=1.60 \pm 0.43 \mathrm{~m}$.

According to the golf project to develop psychological training in line with the needs of athletes themselves, such a large number of studies show that China's psychological training methods for athletes have special targeted and systematic characteristics, which requires long-term and systematic training to achieve remarkable results [5]. Therefore, in order to improve the sports performance of athletes, we should not only improve the physical quality, technical and tactical level, competition awareness, and stress ability of water polo athletes but also cultivate and exercise the psychological ability of athletes.

\section{Experimental Methods}

3.1. Physiological Coherence and Autonomic Balance System. The self-generate physiological coherence system (SPCS) was developed by the Heart Math Institute in the United States. Beijing Baofeng Digital Technology Co., Ltd., independently developed a high-tech biofeedback psychological training product with human-computer interaction, as shown in Table 1 . The system integrates training, testing, and evaluation: above machine feedback project as a means of training, using infrared electric sensors to obtain human heart rate variability signals and the time domain and frequency domain index, the participants can see their heart rate through the computer before and after the autonomic balance, coordination state changes, and after the training heart rate variability charts and spectrum diagram can be obtained directly $[6,7]$. The SPCS system combines heart rate variability and biofeedback effectively and overcomes the dull and rigid deficiencies of previous biofeedback training with its vivid project training and colorful picture effect. At the same time, the constant evaluation feedback also enables the athletes to intuit the changes of their own autonomic nervous system, so as to enhance the psychological adjustment ability of the athletes and better grasp the traditional psychological skills training. The parameters in SPCS specification are presented in Table 2.

See Figure 2 for the usage; 10 college golfers were given SPCS biofeedback training for 4 months. The athletes are 5050 divided into two groups: A and B. Group A athletes have training every Monday and Thursday morning, while Group $\mathrm{B}$ athletes have training every Tuesday and Friday afternoon. Before each formal training, everyone received a basic training, that is, the breathing ball in the SPCS system as a guide to continue to breathe deeply at a consistent rate for 3 minutes. Each person received biofeedback training twice a week [8]. At the end of the experimental intervention, a total of 26 times of training were measured for each person (less than 32 times due to holidays, competitions, and other reasons). Finally, 9 people were selected, and the feedback data of 18 times for each person was considered as effective research data.

In order to ensure that each contestant is tested in the same state, each prefeedback training is based on the adjustment of three minutes. After the adjustment is completed on this basis, the athletes carry out the basic adjustment successively. The computer screen will feedback the adjustment status of the contestants. Among them, the selfregulation states are divided into low, medium, and high, and the colors are red, blue, and green, respectively, so that the athletes can intuitively and vividly understand their current relaxation and regulation state. Each basic adjustment is 3 minutes.

3.2. The Bodhi Tree Project. The Bodhi Tree Project is a training program that applies the three-step method of selfbalancing to train people to quickly enter the coordinated state. The rise and fall of the Bodhi tree are a metaphor for the level of the coordinated state. It helps train the subjects' ability of self-balance regulation and release excessive stress and negative emotions. Training begins with the athlete quickly getting into a steady, coordinated state (which can be 
TABLE 1: Experiment time and place and number of athletes.

\begin{tabular}{llr}
\hline Time & Place & Number of athletes \\
\hline February 23rd to March 26th (in the early) & Golf Lounge & Ten people \\
May 27 to May 15 (mid) & Golf Lounge & Ten people \\
May 16 to June 26 (in the late) & Golf Lounge & Ten people \\
\hline
\end{tabular}

TABLe 2: Parameter description in SPCS instrument manual.

\begin{tabular}{|c|c|c|c|}
\hline $\begin{array}{l}\text { Time domain } \\
\text { data }\end{array}$ & Specific meaning & $\begin{array}{c}\text { Frequency } \\
\text { domain data }\end{array}$ & Specific meaning \\
\hline $\begin{array}{l}\text { M-HRT } \\
(\mathrm{bpm})\end{array}$ & Mean heart rate & $\mathrm{TP}\left(\mathrm{ms}^{2}\right)$ & $\begin{array}{c}\text { The sum of energy less than } 0.4 \mathrm{~Hz} \text { in the frequency } \\
\text { domain of HRV signal }\end{array}$ \\
\hline $\begin{array}{l}\text { SD-HRT } \\
\text { (bpm) }\end{array}$ & Standard deviation of heart rate & $\operatorname{VLF}\left(\mathrm{ms}^{2}\right)$ & $\begin{array}{c}\text { The sum of energy between } 0.0033 \mathrm{~Hz} \text { and } 0.4 \mathrm{~Hz} \text { in the } \\
\text { frequency domain of an } \mathrm{HRV} \text { signal }\end{array}$ \\
\hline SDNN (ms) & The standard deviation of the $\mathrm{N}$ interval & $\mathrm{LF}\left(\mathrm{ms}^{2}\right)$ & $\begin{array}{c}\text { The sum of energy between } 0.04 \mathrm{~Hz} \text { and } 0.15 \mathrm{~Hz} \text { in the } \\
\text { frequency domain of an HRV signal }\end{array}$ \\
\hline $\begin{array}{l}\text { RMS-SD } \\
(\mathrm{ms})\end{array}$ & $\begin{array}{l}\text { The mean of the interval differences } \\
\text { between neighboring NN }\end{array}$ & $\mathrm{LF} / \mathrm{HF}$ & $\begin{array}{l}\text { The energy ratio between the LF frequency band and the } \\
\text { HF frequency band }\end{array}$ \\
\hline SDSD (ms) & $\begin{array}{l}\text { The standard deviation of the interval } \\
\text { difference between adjacent NN }\end{array}$ & LF norm (nu) & $\begin{array}{l}\text { Normalized LF band capability LF } \\
\quad \text { norm }=100 * \text { LF/(Tp-VLF })\end{array}$ \\
\hline PNN50\% & $\begin{array}{l}\text { Percentage of the difference between } \\
\text { adjacent NN intervals greater than } 50 \mathrm{~ms}\end{array}$ & HF norm (nu) & $\begin{array}{l}\text { Normalized LF band capability LF } \\
\text { norm }=100 * \text { LF/(TP-VLF) }\end{array}$ \\
\hline
\end{tabular}

1. Insert the plug of the earpiece information collector and the USB cable into the corresponding slot of the biofeedback sensor

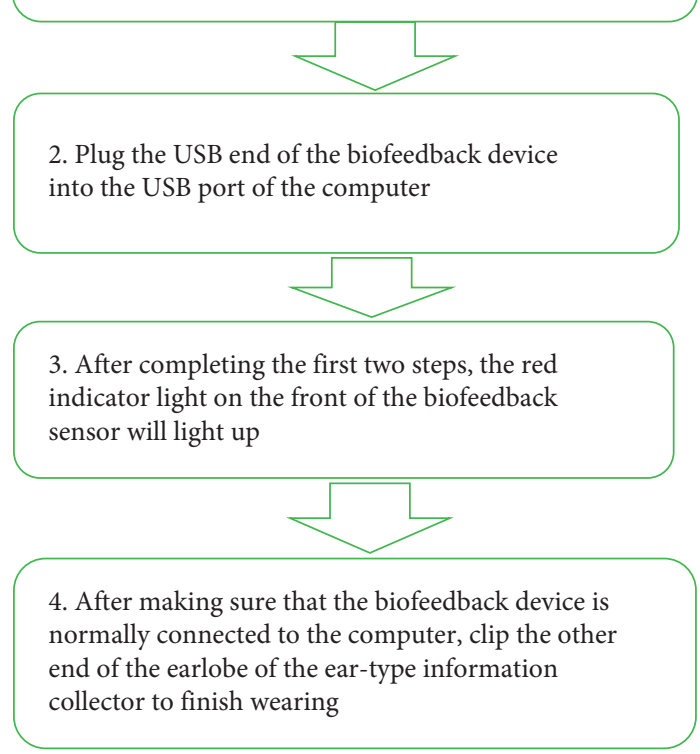

FIgUre 2: Usage.

achieved through deep breathing with a ball in the system) and then maintaining that steady, balanced state. On this basis, the "linden tree" in the computer picture will gradually grow up from the bare surface, and the surrounding environment will gradually turn from dark to colorful, accompanied by quiet and melodious music, all of which "induce" the athletes to slowly enter the best balance state. However, if the athlete's own breathing is disturbed or the stationary state is disturbed, the lush "peepul" will fall back into decay. Therefore, athletes need to maintain such a positive and stable state until the picture shows the success of training. At the same time, athletes should also learn how to calm their emotions and stabilize the breathing rhythm to complete the training under the adverse situation, i.e., the regressive situation of trees.

3.3. Archery Events. Archery project is a training in the environment of external interference and fast focus and eliminates all kinds of interference factors of the game. In the training process, according to the different coordination state, the interference crowd and noise that affect the accuracy of archery will be added around the "competitor." When the coordination state is good, the "player" around no interference, the accuracy of shooting high marks will be improved; when the coordination state is poor, there will be interference crowd around the "competitor," which will lead to the decrease of the accuracy of archery $[9,10]$. This sport is close to golf, where the number of rounds appears immediately after each shot, just as golf knows its score after each hole. Good or bad results may affect the athletes' attention, and in this case, how to focus quickly and finish the next arrow is the ability that athletes must master.

3.4. Dexterity Project. Dexterity: this exercise demonstrates the effects of high coordination on external performance, such as reflexes. In the game, try to operate props to hit small monsters. Under different circumstances, operating props show different reflection speeds. The purpose of this training is to improve the athletes' rapid reaction ability, especially the ability of rapid autonomous balance adjustment under high pressure. When entering the training, the athletes only have 3-5 seconds to prepare to stabilize their emotions and also guide themselves to take an 
effective deep breath according to the breathing balls provided by the system. Then, the computer screen will show small monsters in the random corner, and the athletes need to hit the monsters with the tools. If the athletes miss 5 small monsters, the task will fail. At the same time, if their coordination ability is not stable, the moving speed of props will be slowed down, and the success rate of beating small monsters will be lower, which requires athletes to maintain good autonomous coordination while reacting quickly, that is, they can still maintain stable breathing and strong autonomous coordination ability in the face of stress.

\section{Discussion and Analysis}

4.1. Athletes' Heart Rate. According to the above physiological mechanism principle, the variation trend of athletes' heart rate variability was analyzed from horizontal and vertical aspects, that is, the difference of each index of center rate variability in different event groups and the difference of each index of center rate variability in different time groups. In this way, the changes of autonomic nervous system, sympathetic nervous system, and parasympathetic nervous system of athletes under different project groups and time groups were evaluated.

Bodhi, archery, and psychic dexterity are three interventions with different forms of training feedback. The Bodhi Project mainly focuses on cultivating positive emotions and releasing excessive pressure. Archery mainly trains athletes to focus their attention quickly on an environment of external distractions. Dexterity events mainly cultivate athletes' ability of rapid coordination and selfbalancing under complex environment. Therefore, heart rate variability varies greatly in different events. The changes of HRV in different events and the differences of HRV among different events are analyzed through the data of HRV index parameters feedback, and the influences of different events on athletes' autonomic nervous system are detected. In the SPCS system, training programs are divided into three levels: easy, medium, and hard. Athletes in this study underwent HRV biofeedback training for 4 months. Among them, 4 months are divided into three training periods. The training items of athletes in early training are relatively simple, and the training items of middle training and later training are moderately difficult. The increase of difficulty reflects that the training program requires athletes to improve their ability of self-coordination and balance. Therefore, the HRV of athletes in different training periods will also vary to different degrees. In this study, 11 indicators of the time domain and frequency domain analysis methods were taken as evaluation parameters, and the following analysis and discussion were conducted according to different project groups and time groups.

The mean value of M-HRT in the dexterity items showed an increasing trend, and the values of M-HRT in the dexterity items were higher than those in the Bodhi tree items and the archery items.
4.2. Differences in the Middle Frequency Domain of Variables in Each Group. Tables 3 and 4 further compare LSD multiple mean values for time domain index scores of different projects and different times. The results showed that there was no significant difference in RMS-SD among different training feedback items $(P>0.05)$. There were significant differences in SDNN, RMS-SD, and PNN50 $(P<0.05$, $P<0.01)$, and there was significant difference in SDNN $(P<0.01)$ between dainty and Bodhi tree and archery. According to Table 5, the SDNN value of the dexterity project was significantly lower than that of the linden tree and archery project $(P<0.01)$. In RMS-SD, it was significantly lower than that in linden $(P<0.05)$, and it was significantly lower than that in archery $(P<0.01)$. PNN50 was significantly lower than those of Bodhi tree and archery $(P<0.05)$.

\subsection{Difference Test of Intermediate Frequency Index in Grades} Group. As can be seen from Table 6, indicators VLF, HF, LF/ HF, LF norm, and HF norm have no significant differences among the achievement groups $(P>0.05)$, while indicators $\mathrm{TP}$ and LF have very significant differences among the achievement groups $(P<0.01)$. According to the description, the mean values of indicators TP, LF, and HF in each achievement group all changed the most in the later group, and the mean values in the middle group decreased. The mean values of VLF and HF norm were increasing in each group. The mean values of indicators LF/HF and LF norm showed decreasing changes in each group.

\section{The Results}

In Table 7, under physiological conditions, LF and HF may be possible to represent the intensity of certain autonomic nerve activity, but in cases or under nonphysiological conditions (such as drugs or strenuous exercise), these meanings do not exist. In addition, some scholars also doubt that LF index mainly represents the sympathetic nervous system and believe that LF is more affected by the vagus nerve. Therefore, no matter which value of simple LF and HF is explained, it cannot be explained objectively and scientifically. According to the values of LF/HF, LF norm, and HF norm, we can see that although we cannot prove that the sympathetic and vagus nerves have a significant impact on athlete performance, but the change trend of the indicators and the change trend of average can prove biofeedback training by influencing the athlete's heart rate variability index and positive impact on performance of athletes, while the sympathetic nerve activity of athletes decreases and the parasympathetic nerve activity increases, and the number of athletes who completed the swing decreased. Although these results do not fully support the hypothesis proposed in this study that biofeedback training can significantly improve athletes' athletic performance while improving their heart rate variability, they do indicate that long-term biofeedback training has a positive effect on athletes' exercise tables to a certain extent. 
TABLe 3: Differences of time domain indexes in each group of variables.

\begin{tabular}{|c|c|c|c|c|c|c|}
\hline The independent variable & The dependent variable & Sum of type variance & Degrees of freedom & The mean square & $F$ & Sig \\
\hline \multirow{4}{*}{ Project group } & M-HRT & 103.331 & 2 & 51.666 & 1.095 & 0.340 \\
\hline & SDNN & 5685.205 & 2 & 2842.602 & 14.401 & 0.000 \\
\hline & RMS-SD & 2011.281 & 2 & 1005.640 & 4.951 & 0.010 \\
\hline & PNN50 & 395.987 & 2 & 197.994 & 3.305 & 0.042 \\
\hline \multirow{4}{*}{ Time category } & M-HRT & 86.462 & 2 & 43.231 & 0.917 & 0.405 \\
\hline & SDNN & 4768.753 & 2 & 2384.376 & 12.080 & 0.000 \\
\hline & RMS-SD & 4929.258 & 2 & 2464.629 & 12.134 & 0.000 \\
\hline & PNN50 & 927.601 & 2 & 462.800 & 7.742 & 0.001 \\
\hline \multirow{4}{*}{ Project $*$ time } & M-HRT & 111.915 & 4 & 27.979 & 0.593 & 0.669 \\
\hline & SDNN & 4060.336 & 4 & 1015.084 & 5.143 & 0.001 \\
\hline & RMS-SD & 3495.371 & 4 & 873.843 & 4.302 & 0.004 \\
\hline & PNN50 & 1260.266 & 4 & 315.067 & 5.259 & 0.001 \\
\hline
\end{tabular}

TABLE 4: LSD multiple comparison of the mean values of time domain indicators in each project group.

\begin{tabular}{|c|c|c|c|c|c|}
\hline The dependent variable & Project & Group & Mean difference & Std. error & Sig \\
\hline \multirow{3}{*}{ SDNN } & 1 & 3 & -1.625 & 3.824 & 0.672 \\
\hline & 1 & 3 & 16.904 & 3.824 & 0.000 \\
\hline & 2 & 3 & 18.529 & 3.824 & 0.000 \\
\hline \multirow{3}{*}{ RMS-SD } & 1 & 2 & -1.944 & 3.879 & 0.618 \\
\hline & 1 & 3 & 9.464 & 3.879 & 0.017 \\
\hline & 2 & 3 & 11.408 & 3.879 & 0.004 \\
\hline \multirow{3}{*}{ PNN50 } & 1 & 2 & 0.602 & 2.107 & 0.776 \\
\hline & 1 & 3 & 4.962 & 2.107 & 0.021 \\
\hline & 2 & 3 & 4.360 & 2.107 & 0.042 \\
\hline
\end{tabular}

TABLE 5: Data of three games.

\begin{tabular}{lcccc}
\hline The time domain index & Training feedback program & The early stage of the training & Medium-term training & The late training \\
\hline \multirow{3}{*}{ M-HRT } & Banyan tree & $70.572 \pm 8.577$ & $73.731 \pm 6.404$ & $68.846 \pm 6.299$ \\
& Archery & $72.076 \pm 7.518$ & $73.813 \pm 6.958$ & $69.852 \pm 5.317$ \\
& Ingenuity & $73.029 \pm 8.759$ & $73.386 \pm 6.104$ & $74.858 \pm 4.797$ \\
\hline \multirow{3}{*}{ SDNN } & Banyan tree & $84.223 \pm 12.786$ & $76.203 \pm 13.382$ & $106.992 \pm 15.196$ \\
& Archery & $89.602 \pm 18.068$ & $76.583 \pm 12.784$ & $106.108 \pm 17.210$ \\
& Ingenuity & $76.294 \pm 11.235$ & $72.226 \pm 15.319$ & $68.187 \pm 7.479$ \\
\hline \multirow{3}{*}{ RMS-SD } & Banyan tree & $58.635 \pm 11.109$ & $50.501 \pm 12.880$ & $80.876 \pm 16.251$ \\
& Archery & $62.811 \pm 18.312$ & $52.304 \pm 10.823$ & $80.729 \pm 20.478$ \\
& Ingenuity & $58.115 \pm 10.615$ & $52.603 \pm 14.624$ & $50.903 \pm 8.845$ \\
\hline \multirow{3}{*}{ PNN50 } & Banyan tree & $30.478 \pm 5.216$ & $24.233 \pm 8.170$ & $40.454 \pm 9.018$ \\
& Archery & $31.086 \pm 9.461$ & $24.406 \pm 8.112$ & $37.867 \pm 6.444$ \\
& Ingenuity & $28.891 \pm 79.60$ & $28.123 \pm 9.029$ & $23.264 \pm 4.699$ \\
\hline
\end{tabular}

TABLE 6: Description and difference comparison of each frequency domain index in the achievement group.

\begin{tabular}{|c|c|c|c|c|}
\hline Frequency domain index & Results group & $\mathrm{M} \pm \mathrm{SD}$ & $F$ & Significant \\
\hline $\mathrm{TP}$ & $\begin{array}{c}\text { In the early } \\
\text { Mid } \\
\text { In the late } \\
\end{array}$ & $\begin{array}{c}1076.77 \pm 306.89 \\
728.12 \pm 216.67 \\
1541.22 \pm 453.53 \\
\end{array}$ & 12.95 & 0.00 \\
\hline VLF & $\begin{array}{c}\text { In the early } \\
\text { Mid } \\
\text { In the late }\end{array}$ & $\begin{array}{c}64.48 \pm 36.48 \\
71.53 \pm 38.17 \\
141.39 \pm 117.70 \\
\end{array}$ & 2.93 & 0.07 \\
\hline LF & $\begin{array}{c}\text { In the early } \\
\text { Mid } \\
\text { In the late }\end{array}$ & $\begin{array}{c}942.53 \pm 255.47 \\
618.62 \pm 197.73 \\
1301.31 \pm 335.85\end{array}$ & 14.49 & 0.00 \\
\hline
\end{tabular}


TABLE 6: Continued.

\begin{tabular}{|c|c|c|c|c|}
\hline Frequency domain index & Results group & $\mathrm{M} \pm \mathrm{SD}$ & $F$ & Significant \\
\hline $\mathrm{HF}$ & $\begin{array}{c}\text { In the early } \\
\text { Mid } \\
\text { In the late }\end{array}$ & $\begin{array}{l}69.75 \pm 86.50 \\
37.96 \pm 27.89 \\
98.51 \pm 72.87 \\
\end{array}$ & 1.82 & 0.18 \\
\hline $\mathrm{LF} / \mathrm{HF}$ & $\begin{array}{l}\text { In the early } \\
\text { Mid } \\
\text { In the late }\end{array}$ & $\begin{array}{c}39.12 \pm 23.50 \\
28.91 \pm 7.60 \\
27.00 \pm 7.00\end{array}$ & 0.68 & 0.36 \\
\hline LF norm & $\begin{array}{l}\text { In the early } \\
\text { Mid } \\
\text { In the late }\end{array}$ & $\begin{array}{c}94.58 \pm 3.35 \\
93.92 \pm 3.42 \\
92.39 \pm 5.6 \\
\end{array}$ & 0.61 & 0.54 \\
\hline HF norm & $\begin{array}{c}\text { In the early } \\
\text { Mid } \\
\text { In the late }\end{array}$ & $\begin{array}{l}5.41 \pm 3.35 \\
6.07 \pm 3.42 \\
7.60 \pm 569\end{array}$ & 0.68 & 0.36 \\
\hline
\end{tabular}

TABLE 7: LSD multiple comparison of median frequency index mean of each achievement group.

\begin{tabular}{lcccr}
\hline The dependent variable & Project & Group & Mean difference & Std. error \\
\hline \multirow{2}{*}{ TP } & 1 & 2 & 348.65 & 160.28 \\
& 1 & 3 & -464.44 & 160.28 \\
\hline \multirow{2}{*}{ LF } & 2 & 3 & -813.10 & 0.04 \\
& 1 & 2 & 323.91 & 0.00 \\
& 1 & 3 & -358.77 & 0.00 \\
\hline
\end{tabular}

\section{Conclusions}

The biofeedback training effect of athletes in the first stage is not obvious, but gradually increases with the deepening of the training process. When the training goes to the third stage, the training effect is the most obvious after the difficulty is increased again. Biofeedback relaxation training can help athletes improve the balance of sympathetic and parasympathetic nerves, effectively inhibit the activity of sympathetic nerves, enhance tension, effectively increase the variability of athletes' heart rate, and strengthen the ability of psychological relaxation. Try to test the athletes when they participate in the competition to observe whether the athletes' heart rate variability changes during training and competition and how the trend of change is. In this way, the effect of psychological training on athletes can be examined more comprehensively. At the same time, the experimental results indicate that longterm biofeedback training can effectively balance the sympathetic and parasympathetic nervous systems of athletes, significantly enhance the autonomic nervous regulation ability of athletes, and increase the vagal tension and heart rate variability of athletes. Some research results show that biofeedback training can enhance and improve athletic performance of athletes to a certain extent.

\section{Data Availability}

The data used to support the findings of this study are available from the corresponding author upon request.

\section{Conflicts of Interest}

The authors declare no conflicts of interest.

\section{References}

[1] S. Zhao, D. Yu, Y. Zou et al., "Integration of CMOS image sensor and microwell array using 3-D WLCSP technology for biodetector application," IEEE Transactions on Components, Packaging, and Manufacturing Technology, vol. 9, no. 4, pp. 624-632, 2019.

[2] N. Sun and H. Li, "Super resolution reconstruction of images based on interpolation and full convolutional neural network and application in medical fields," IEEE Access, vol. 99, p. 1, 2019.

[3] P. Caputo, M. Porto, R. Angelico, V. Loise, P. Calandra, and C. Oliviero Rossi, "Bitumen and asphalt concrete modified by nanometer-sized particles: basic concepts, the state of the art and future perspectives of the nanoscale approach," Advances in Colloid and Interface Science, vol. 285, no. 275, Article ID 102283, 2020.

[4] D. Van Opdenbosch and C. Zollfrank, "Modeling the compressive behavior of anisotropic, nanometer-scale structured silica," Advanced Engineering Materials, vol. 21, no. 6, Article ID 1801097, 2019.

[5] K. K. Sahoo, P. Sarkar, and R. Davis, "Mechanical properties of silica fume concrete designed as per construction practice," Proceedings of the Institution of Civil Engineers-Construction Materials, vol. 172, no. 1, pp. 20-28, 2019.

[6] K. He, C. Ye, Y. Deng et al., "Study on the microscale structure and anti-seepage properties of plastic concrete for cut-off walls modified with silica fume: experiment and modelling," Construction and Building Materials, vol. 261, no. 2-3, Article ID 120489, 2020.

[7] J. Wei, B. Gencturk, A. Jain, and M. Hanifehzadeh, "Mitigating alkali-silica reaction induced concrete degradation through cement substitution by metakaolin and bentonite," Applied Clay Science, vol. 182, pp. 105257.1-105257.14, 2019.

[8] T. Inose, T. Oikawa, M. Tokunaga et al., "Development of composite nanoparticles composed of silica-coated nanorods 
and single nanometer-sized gold particles toward a novel X-ray contrast agent," Materials Science and Engineering B, vol. 262, no. 11, Article ID 114716, 2020.

[9] S. Y. Duan, Z. X. Wu, J. Y. Piao, and D. Bin, "Controlling the reaction kinetics in solution for uniform nanoshells of metal sulfides with sub-nanometer accuracy," Science Bulletin, vol. 64 , no. 4, pp. 232-235, 2019.

[10] E. Goudeli, J. Lee, and C. J. Hogan, "Silica nanocluster binding rate coefficients from molecular dynamics trajectory calculations," Journal of Aerosol Science, vol. 146, no. 1, Article ID 105558, 2020. 